\title{
Predicting Remaining Life of Transmission Tower Steelwork Components
}

\author{
M. Segovia \& V. M. Catterson \\ University of Strathclyde, Glasgow, United Kingdom
}

A. Stuart, L. Johnston, H. Bain \& R. McPhaden

Scottish and Southern Energy Power Distribution, Perth, United Kingdom

R. Wylie \& A. Hernandez

Scottish Power Energy Networks, Blantyre, United Kingdom

\begin{abstract}
Failures in transmission tower's components usually result in extended disruption of power supply. Repair is very costly as it involves replacement of the transmission lines' sections affected. Additionally, it might also entail litigation cost associated with power disruption. Maintenance decisions have to be taken in time to prevent a failure. At present, maintenance decisions are mainly based on expert's judgement, who perform inspections every 10 to 12 years.

On specific sites, tower's components degrade much faster due to aggressive atmospheric conditions, with corrosion being the primary cause of deterioration. In this context, data indicating health state from an UK utility were used to create a Cox model that relates the time before a failure occurs to climatic and atmospheric conditions highly correlated with corrosion. The paper demonstrates the use of the model for predicting remaining tower life, and highlights how this can feed into maintenance planning.
\end{abstract}

\section{INTRODUCTION}

Transmission towers are the steel-framed structures supporting overhead power lines, which constitute a significant portion of the electricity transmission grid. While transmission towers and associated components are generally highly reliable, if a failure takes place it usually results in extended disruption of power supply. Repair is very costly: it involves replacement of transmission lines' sections affected. Additionally it might also entail litigation cost associated with power disruption.

Appropriate maintenance decisions have to be taken in time in order to prevent such a failure. At present, condition assessment and maintenance decisions are mainly based on the judgement of experts, who perform inspections and assess component condition on site once every 10 to 12 years. Furthermore, information captured during inspections may not be detailed or objective enough to ensure a high level of confidence in asset condition, leading to conservative replacements occurring potentially before assets are at risk of failure.

The inspection time is based on lifetime expectancy, which varies between 60 and 80 years. However, it has been observed that on specific sites, tower components degrade much faster due to aggressive atmospheric conditions in the area, with corrosion being the primary cause of deterioration and a long term failure mode, i.e. icing may cause catastrophic failure, but does not have a long term effect.

In this context, this paper presents a statistical model that considers climatic and atmospheric conditions related to corrosion for estimating tower steelwork component remaining life, so that preventive maintenance actions can be planned in advance. The model is a Cox survival model (Cox, 1972) which strives to relate the time before an event happens to one or more covariates related to the event. In this case the event is the failure of a steelwork component and the covariates are the climatic and atmospheric conditions.

Many parameters influence deterioration due to corrosion. According to the literature, the main environmental factors involved in the corrosion process are humidity, temperature, pollution, and airborne chlorides (ISO 9223, 2012) although other variables are suspected of having an influence on the process too. In this paper, data indicating health state from in-service towers from a UK utility as well as data on environmental factors on site have been gathered. Firstly, a correlation study was performed in order to identify the factors that have the strongest influence on the corrosion phenomenon. The results of this study are presented in this paper. Secondly, the variables presenting the highest correlations were used to construct a statistical model to predict remaining life. A Cox model was chosen due to the nature of the data on health state gathered. The paper demonstrates the use of the model for predicting 
remaining tower life, and highlights how this can feed into maintenance planning.

The paper is organised as follows: Section 2 provides an overview of corrosion models in the literature. Section 3 describes the data gathered for the study. Section 4 introduces the Cox model and justifies the selection. Section 5 displays the results of the correlation analysis to identify variables highly related to the corrosion process. Section 6 shows the application of the Cox model, and Section 7 provides some conclusions and future work.

\section{CORROSION MODELS: CLASSIFICATION SCHEMES, STATISTICAL MODELS AND OTHERS}

The main aim of corrosion models in the literature is to predict the extent of corrosion loss at a specific location and time as a function of the environmental factors involved in the corrosion process and the attributes of the material being analysed (Straub and Faber, 2007).

However, the majority of the models focus on estimating the rate of corrosion on specific sites considering environmental factors. The obtained value is then utilised to predict the extent of corrosion frequently through a power law:

$$
D(t)=A t^{b}
$$

where $D(t)$ represents the corrosion depth for the considered time interval given in micrometres $(\mu \mathrm{m})$, or as a mass in grams per square metre. $A$ and $b$ are constants, $A$ being the corrosion rate and $t$ the time in service. However, it has been noted in the literature that the power law does not always fit the data well, moreover, this law does not account for significant changes in the values of $A$ or $b$ in the long term (Melchers, 2009). Furthermore, because of the formation of corrosion products on the metal surface, the initial corrosion rate usually decreases over a long-term period and is not constant.

Roberge et al. (2002) classify the corrosion models in the literature in three categories: classification schemes, statistical models, and other models.

Within classification schemes ISO 9223 (2012) provides a comprehensive classification system for the corrosivity of the atmospheres where the metallic structures are located. The atmospheric corrosivity is divided into six categories, ranging from $\mathrm{C} 1$ (very low) to C5 (very high) and CX (extreme). The determination of the corrosivity category can be done either by measurement of the corrosion loss in the first year of exposure of the structure or, if this is not possible, by information on environmental condition and exposure. The key environmental factors to determine the atmosphere corrosivity are the temperature-humidity complex, the atmospheric salinity, and the pollution concentration. The rate of atmospheric corrosion, $A$ in equation (1), can be estimated from the corrosivity category.

Another example of a classification scheme is given by the PACER LIME algorithm (1980), where an environmental corrosivity scale was developed for maintenance planning of aircraft structures. The corrosivity category was obtained considering distance from salt water, moisture factors, and pollutant concentrations. In spite of the fact that both schemes are straightforward methods, they are limited in their precision as they do not consider localised mechanisms of corrosion, such as pitting or wind effects.

Statistical models in the literature have been demonstrated to be effective in areas where data are gathered, but limited when estimating corrosion rate in areas with different characteristics, or when available corrosion information is highly non-linear (Kenny et al., 2009).

Among others, the International Organisation for Standardisation (ISO) examined eight years of atmospheric corrosion data on different types of metals in up to 51 different sites to develop a regression model for calculating the corrosion rate (Dean and Reiser, 2002). The regression model considered the temperature, the relative humidity, the atmospheric salinity and the pollution concentration. The results are utilised by the standard ISO 9224 to obtain $b$ in equation (1), this standard is normally used in conjunction with ISO 9223.

Similarly to the regression model developed in Dean and Reiser (2002), the International Cooperative Program on Effects on Materials (ICP Materials) (Tidblad et al., 2000) and The Iberoamerican Atmospheric Corrosion Map project (MICAT) (Feliu et al., 1993) developed two regression models for the estimation of corrosion rates by considering data from different sites and different types of metals.

Beyond these regression models, other authors have utilised artificial neural networks to estimate and predict the rate of corrosion. These models are also based on experimental data from metal exposed to outdoor atmospheres in different sites, see Kenny et al. (2009)) and Pintos et al. (2000). As in the case of regression models, artificial neural networks have to be retrained when estimating corrosion rates in different areas and environmental contexts.

The final category of models considered, other models, corresponds to those models that try to explain the mechanics of the corrosion, e.g. marine aerosol transport and deposition, or wind effects on local corrosivity.

Feliu et al. (1999) assume the existence of a steady source of marine aerosol particles and investigate how these aerosols are deposited and where. The deposition rate is characterized by the deposition velocity which depends on the particle size, the surface roughness, and the wind speed. This model is applicable within the first few hundred meters 
from the aerosol's source, but after that other factors, such as rain, affect the chloride concentration.

In Klassen and Roberge (2001) the effect of the wind on atmospheric corrosivity was modelled by exposing samples located in different degrees of shelter from the wind near a highway. A $42 \%$ reduction in average corrosivity was observed between protected and unprotected samples. Additionally, Lyon et al. (1995) investigates the corrosion process within a droplet, and Hayne (1988) studies how pollutants are transferred to metallic surfaces.

\section{THE DATA}

Data from a condition survey of 1543 towers from 30 different circuits were provided by a UK utility. From this survey data, only the steel tower components have been considered, as the main focus of this work was to estimate the remaining life of tower components affected by corrosion. Component condition was assessed through visual inspection and is given on a scale from 1 to 4 , with 4 being the worst condition.

Starting with this data, a database was built. The list of variables included in the database is described below. Information on variables in categories 1 to 4 was provided by the utility. The rest of the variables refer to environmental factors on site which were selected considering literature review and discussions with overhead line experts. Data for these environmental factors was gathered through online sources, including the UK Meteorological Institute (Met Office), the Department of Energy and Climate Change (DECC), and online maps.

1. Location (given in GPS coordinates),

2. Condition assessment of tower components: Tower and crossarm alignments, tower legs, step bolts, bracing, crossarms, peak, paintwork/galvanising, flagsocket/step mask.

3. Time since maintenance, which could indicate time since a component was replaced or since the tower was painted. In general this variable gives an indication of the age of the component.

4. Time in service.

5. Minimum distance from corrosion source. This variable is used as a proxy variable to estimate the pollutant concentration on site, in particular to estimate the concentration of $\mathrm{SO}_{2}$. The main reason to consider this variable is that information on pollutant concentration was not found through online sources. The considered sources of $\mathrm{SO}_{2}$ are: nearest main road, mines, industrial areas, cities and power stations. This variable is measured in metres $(m)$.

6. Distance from the sea. This is a proxy variable given in metres $(m)$ used to estimate the concentration of airborne salinity.

7. Elevation, measured in metres $(m)$.
8. Distance from closest wind farm. This variable is measured in metres $(m)$.

9. Wind speed. Records of annual average wind speed were found for weather stations close to the tower sites. This variable is measured in kilometres per hour $(\mathrm{km} / \mathrm{h})$.

10. Extremes of temperature. This variable contains the mean of the daily difference between maximum and minimum ambient temperatures over all years the tower is in service.

\section{THE COX MODEL}

\subsection{Model background}

The Cox regression model is a survival analysis model (Cox, 1972). This type of model strives to relate the time before an event happens (in this case a failure of a transmission tower steelwork component), to one or more covariates (explanatory variables) related to the event. The Cox model estimates survival curves when considering several covariates simultaneously, e.g. temperature, pollution concentration, airborne salinity concentration, etc.

This model provides the expression of the risk at a given time $t$ for any individual with a given specification of a set of covariates (Kleinbaum and Klein, 2005). The risk at time $t$ is given by the product of two quantities, the baseline hazard function $\left(h_{0}(t)\right)$ which represents the common risk to all the individuals under study, and the effect of the covariates on the risk, which is given by the exponential of the linear combination of the covariates. The expression of the hazard is shown below:

$h(t)=h_{0}(t) e^{\sum \beta_{i} X_{i}}$

where $X_{i}, i=1, \ldots, p$, represents the covariates and $\beta_{i}$ represents the effect of the covariate on the risk of the event of interest happening, in this case the failure of a steelwork component.

The baseline function depends on $t$, and the covariates are time-independent in this expression. Nevertheless it is possible to consider timedependent covariates in the Cox model. Additionally, the baseline function can be replaced by a given function if it is known, e.g. the Weibull distribution. However it is possible to work with an unspecified baseline function in a Cox model and still produce reasonably good estimates of the survival curves and the hazard function (Kleinbaum and Klein, 2005).

The hazard function can be converted to a corresponding survival function, which is the basis for determining the adjusted survival curves. The expression of the survival function in a Cox model is:

$$
S(t)=\left[S_{0}(t)\right]^{\sum \beta_{i} x_{i}}
$$

where $S_{0}(t)$ is the baseline survival function.

Typically, when computing survival curves, the value chosen for a covariate being adjusted is an av- 
erage value, in particular the mean. Therefore, the estimation of the survival function is done considering the mean of the covariate, and the general expression for this estimation is the following:

$$
\hat{S}(t)=\left[\hat{S}_{0}(t)\right]^{e^{\sum \hat{\beta}_{i} \bar{x}_{i}}}
$$

where $\hat{S}(t), \hat{S}_{0}(t)$ and $\hat{\beta}_{i}$ are the estimates of the survival function, the baseline survival function, and the hazard ratios respectively. Additionally $\bar{X}_{i}$ represents the mean of the ith covariate. Alternatively, it is possible to obtain a survival curve for a particular individual by providing the specific values of the covariates for that individual.

\subsection{Rationale for the selection of the Cox model}

As described in Section 2, in the literature a number of models used for prediction of corrosion progress are linear regression models. This type of model generally tries to estimate the rate of corrosion considering environmental conditions on site.

However, data gathered during this project are not suitable for the development of a linear regression model, as the condition data available is a health index ranking between 1 and 4, not a measurement of depth of metal lost over time.

Unlike linear regression models, the outcome of the Cox regression model is the time to an event of interest, in this case the failure of the steelwork component. Moreover, the Cox model uses failure times as well as censored failure times on the individuals of interest. Censored failure times refer to observed survival times that are shorter than the time to failure for those components, i.e. the true failure time is unknown because the last time the components were observed they were still operative.

Additionally, the Cox model is a semi-parametric model, where the baseline hazard function does not need to be specified if it is unknown. Even though the baseline hazard is not specified, the Cox model is a robust model that will closely approximate the results obtained with the correct parametric form followed by the data, e.g. if the data follow a Weibull distribution, the Cox model will give results comparable to those obtained using a Weibull model (Kleinbaum and Klein, 2005). In this particular study the baseline hazard could have been obtained through the standards ISO 9223 (2012) and 9224 (2012), however the data gathered for this project do not allow the use of the equations presented in those standards. To apply these equations, metal loss measurements and other environmental information such as pollution concentration and airborne chlorides concentration are needed. However proxy variables are used instead for the environmental parameters as precise measurements for these two variables could not be collected.

\section{CORRELATION ANALYSIS}

A correlation study was performed to select the environmental factors with the highest influence on the corrosion process.

The variables described in Section 3 can be considered as two categories: those indicating component condition, i.e. category 2; and those thought to have an influence on the corrosion process, i.e. categories 3 to 10 . The aim is to investigate the level of influence that each variable in the second group has on the condition variables in the first group, i.e. how much the level of corrosion in a component can be attributed to an influencing variable. The variables with the strongest influence were selected to be covariates of the Cox model.

\subsection{Kendall correlation}

As a first step towards the selection of the covariates, the Kendall correlation coefficients between the variables indicating component condition and the environmental factors were obtained. The Kendall correlation coefficient was selected because it can be used with discrete and continuous variables and does not require that the variables follow a Normal distribution (Corder and Foreman, 2009). This coefficient provides a value between -1 and 1 . However its values are not based on a linear scale, therefore the interpretation of the strength of the relationship between two variables is not straightforward, e.g. a coefficient of 0.6 does not indicates a relationship twice as strong as when the coefficient is 0.3 . Consequently, it is important to also test if any relationship is significant, i.e. strong enough to be considered a true result.

The values for the Kendall correlation coefficient for the different pairs of variables are not shown in this paper due to space limitations, although the main results are provided:

- In general, there are no very strong correlations. The strongest correlation factor is -0.351 , with the smallest statistically significant correlation factor being -0.062 . This suggests there is no single factor which dominates the corrosion process.

- The variable extremes of temperature seems to present the strongest relationship with component condition. In all cases this relationship is direct, i.e. when the average difference between daily minimum and maximum temperature increases, the deterioration of steelwork components increases.

- Minimum distance from corrosion source has in in general a direct relationship with the deterioration state of the components, i.e. when the distance from the corrosion source increases the deterioration state also increases. This was an unexpected result, given that this variable is used as a proxy for the pollution concentration ( $\mathrm{SO}_{2}$ concentration) which is known to increase corrosion. 
- Distance from the sea has a direct relationship in most cases. This is also an unexpected result. However the correlation is weak in many cases.

- Average wind speed shows an inverse relationship with the deterioration state in most cases, i.e. in areas with lower wind speeds the deterioration state is higher. A possible explanation could be that water and pollutants will be deposited and remain on the tower surface for longer at reduced wind speeds.

Considering these results and taking into account that in the literature the variables temperature, humidity, pollution concentration, airborne chlorides concentration, and wind speed/direction are identified as the main factors influencing the corrosion process, it was decided to include temperature and wind speed as covariates in the Cox model. Wind direction was not considered, as information about this variable could not be gathered through online sources. Additionally it was decided to carry out further analysis for the following variables: minimum distance from corrosion source as a proxy variable of pollution concentration, distance from the sea as a proxy variable of airborne chlorides concentration, and magnetic fields. The latter one was suggested during discussion with overhead line experts.

\subsection{Correlation between minimum distance from corrosion source and component condition}

Results from the Kendall correlation coefficient in section 5.1 were unexpected, as the variable minimum distance from corrosion source seems to have a direct relationship with the deterioration state of the steelwork tower components. It was decided to investigate if the age had an impact on the results.

Figure 2 displays the scatter plot of the variable time since maintenance, which gives an indication of the age of the components, and the variable minimum distance from a corrosion source.

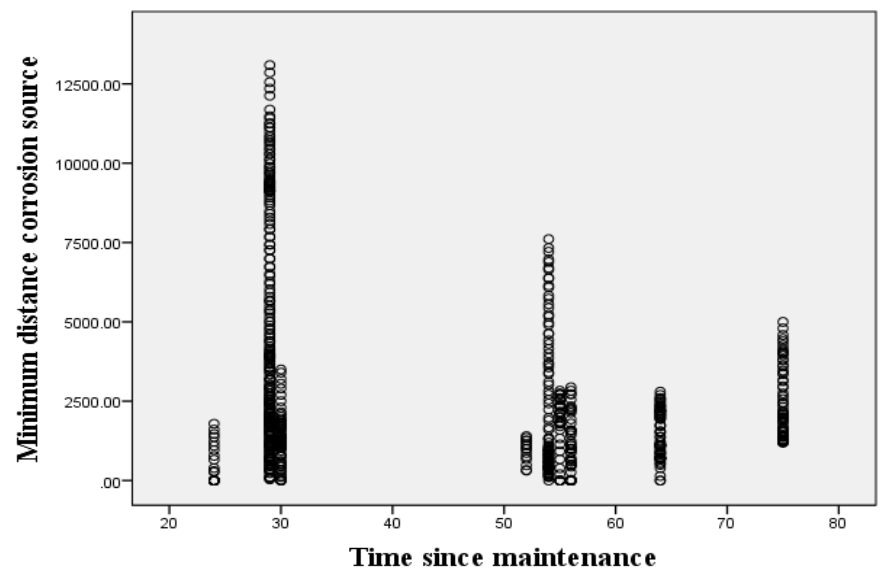

Figure 1. Scatter plot of time since maintenance/distance from corrosion source

Figure 1 shows that older and newer components are situated at different distances from corrosion sources. The oldest components are situated at a maximum distance of $5 \mathrm{~km}$, however components around 30 years of age can be located anywhere be- tween $0 \mathrm{~km}$ and $13 \mathrm{~km}$. Moreover, the Kendall correlation coefficient value for these two variables is -0.130 , and it is a significant relationship (see section 5.1), consequently newer components are situated at larger distances and vice versa. In summary, the age of the components does not seem to have an effect on the results of the correlation analysis.

Taking into account the results, it was decided to discard this variable as it did not provide a good indication of the pollution concentration around the area where towers are located.

\subsection{Correlation between distance from the sea and component condition}

Bearing in mind the results described in section 5.1, the potential impact of the age of the components on the result for the variable distance from the sea was investigated as well. Figure 2 displays the scatter plot of time since maintenance and distance from the sea.

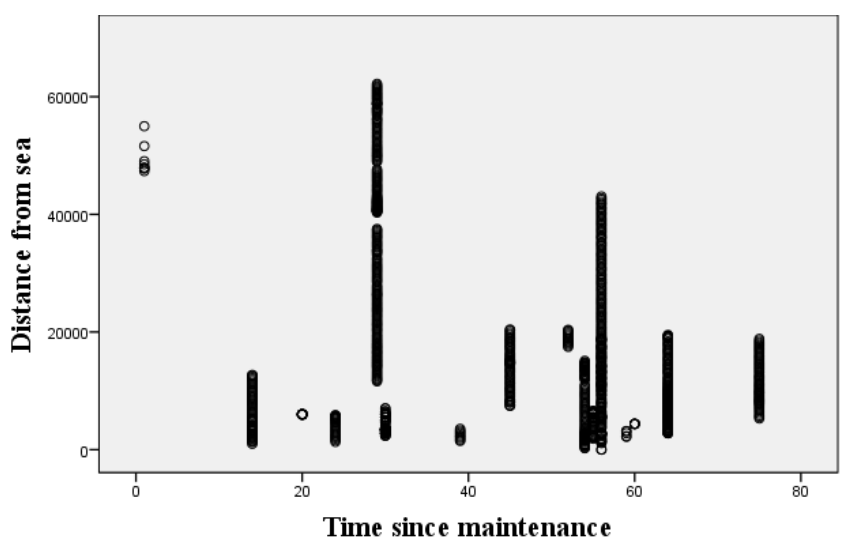

Figure 2. Scatter plot of time since maintenance/distance from the sea

Similarly to the results in section 5.2, this figure shows that older and newer components are situated at different distances from the sea. In fact, components 60 years of age or older are situated less than $20 \mathrm{~km}$ from the sea, but also components around 18 years of age. The results of the Kendall correlation coefficient, -0.104 , show an indirect relationship between these two variables, and it is a significant relationship. Therefore newer components are situated at larger distances and vice versa. Hence, the age of the components is not influencing the results of the correlation analysis for the variable distance from the sea. Accordingly, this variable was discarded as it did not provide a good indication of the airborne chloride concentration around the area where towers are located.

\subsection{Correlation between magnetic fields and component condition}

The magnetic field in the vicinity of overhead line conductors depends on the conductor geometry, and current flowing in the conductor. Figure 3 shows 
typical magnetic fields for different overhead lines, which have been sourced from the EMFS website (http://www.emfs.info/). The magnetic field decreases with the distance from the centre of the conductor

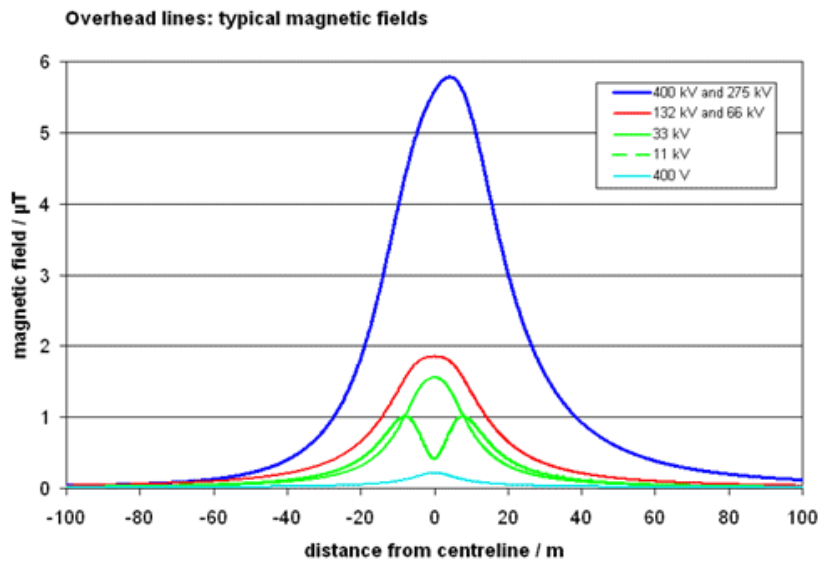

Figure 3. Typical magnetic fields for overhead lines (source EMFS)

Considering this information, tower components were divided into three groups based on their distance to the conductor with the aim of investigating if there is a relationship between magnetic field and component condition. The three groups are described below:

- Group 1 (distance from the centre of the conductor grouping 0 to $5 \mathrm{~m}$ ) comprises left and right circuit components and crossarms.

- Group 2 (distance from the centre of the conductor grouping 5 to $8 \mathrm{~m}$ ) refers to the peak.

- Group 3 (distance from the centre of the conductor grouping more than $8 \mathrm{~m}$ ) refers to tower legs

Only components with similar age were considered to determine if the magnetic field has an impact on the component deterioration. To compare the deterioration of the components in different magnetic field groups, a Kruskal-Wallis test was performed. The Kruskal-Wallis test is the non-parametric version of the ANOVA. The reason for utilising this test is that the variables are not normally distributed. This test contrasts the hypotheses:

$\mathrm{H}_{0}$ : The medians of the groups are the same

$\mathrm{H}_{1}$ : The medians of the groups are different

The p-value of this contrast is 0.372 , which is greater than $\alpha=0.05$ (significance level). Therefore, there are no significant differences in the deterioration of the components belonging to different magnetic fields groups.

In spite of this result, after discussions with overhead line experts, it was decided to include this variable as a covariate in the Cox model due to its potential for impact on steelwork deterioration.

\section{COX MODEL FOR TOWER COMPONENTS}

For the fitting of the Cox model to the data the following variables are introduced:
1. Vector of covariates. This vector contains data on the variables wind speed, magnetic fields and the variable extremes of temperature.

2 . The time since maintenance.

3. A variable containing $0 \mathrm{~s}$ or $1 \mathrm{~s}$ depending on if the component is in deterioration state 4 or in deterioration states 1 to 3 . It is considered that state 4 represents the failure of the component, while states 1 to 3 are transitional states towards the failure. Therefore, the value 0 of the variable corresponds to the failure of the component, and the value 1 indicates that the component has not reached the end of its life. The age of the latter components is hence regarded as a censored time, given that the failure time is not available.

It should also be noted that the present paper considers data which represents a snapshot of the state of the components at a given moment in time. Timeindependent covariates are therefore more appropriate. Therefore, equations (2) - (4) are applied in this section.

Table 1 displays the estimation of the parameters $\beta_{\mathrm{i}}$, as well as the exponential of the estimation. The exponential of these values gives an indication of the increase in failure hazard per unit of increase in the correspondent covariate.

Table 1. Estimation of the effect of the covariates

\begin{tabular}{lcc}
\hline Covariate & $\hat{\beta}_{i}$ & $\operatorname{Exp}\left(\hat{\beta}_{i}\right)$ \\
\hline Extreme of temp. & 0.9111 & 2.4870 \\
Wind speed & -0.1224 & 0.8848 \\
Magnetic fields & -0.1314 & 0.8769 \\
\hline
\end{tabular}

The biggest contributor to failure hazard rate is the variable extremes of temperature. This result agrees with the correlation study in section 5 . The failure hazard will increase by 2.4870 times per $1^{\circ} \mathrm{C}$ increase in the range of temperatures experienced by the component in a day, considering annual averages.

If wind speed is considered, the value in Table 1 indicates that when the average of wind speed increases by $1 \mathrm{~km} / \mathrm{h}$, on an annual basis, the failure hazard will decrease by 0.8848 times. This result also agrees with the previous correlation study.

Finally, the value for the variable magnetic fields implies that the failure hazard decreases by 0.8769 times when the distance from the conductor grouping increases. Although the results in the previous section show no differences between the magnetic field groups in terms of average deterioration, the estimated values in Table 1 shows that magnetic fields do have an effect on the failure hazard, justifying its inclusion in the model.

Figure 4 presents the estimated Survival function (see equation (4)) for the whole sample of circuits and steelwork components for the covariates' means. 


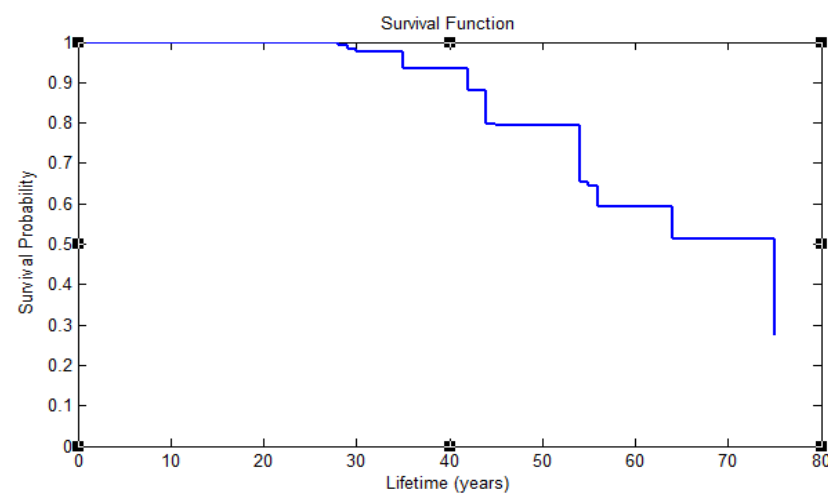

Figure 4. Survival function for the sample

The survival function is plotted as a step function. This is due to the semi-parametric nature of the Cox model where the expression of the baseline hazard is unknown and it is just estimated at points in time where one or more failures have occurred. Therefore, between failure times, the survival probability stays constant. At failure times the survival probability is obtained by taking into account the number of failures at that instant of time.

It is also possible to obtain the survival curve for a particular component/circuit with the Cox model. For that purpose it is necessary to estimate the baseline cumulative hazard function, i.e. $\mathrm{H}_{0}(\mathrm{t})$, the parameters $\beta_{\mathrm{i}}$, and introduce the values of the covariates $X_{i}$ for the particular individual in equation (4), in this case a component in a specific tower. As an example, let us suppose that a tower is located in an area were the variables of interest have the following values: Annual average of extremes of temperature: $6.5^{\circ} \mathrm{C}$, annual average wind speed: $16.2 \mathrm{~km} / \mathrm{h}$. Let us also consider the crossarms within this tower, which are located at a distance between 0 and $5 \mathrm{~m}$ from the conductor grouping, therefore the value for the variable magnetic fields is 1 . The expression of the survival curve for this particular component is obtained from (4) and is given by:

$$
\hat{S}(t)=\left[\exp \left(-\hat{H}_{0}(t)\right)\right]^{2.4870 * 6.5+0.8848 * 16.2+0.8769 * 1}
$$

Figure 5 displays the survival curve for this component:

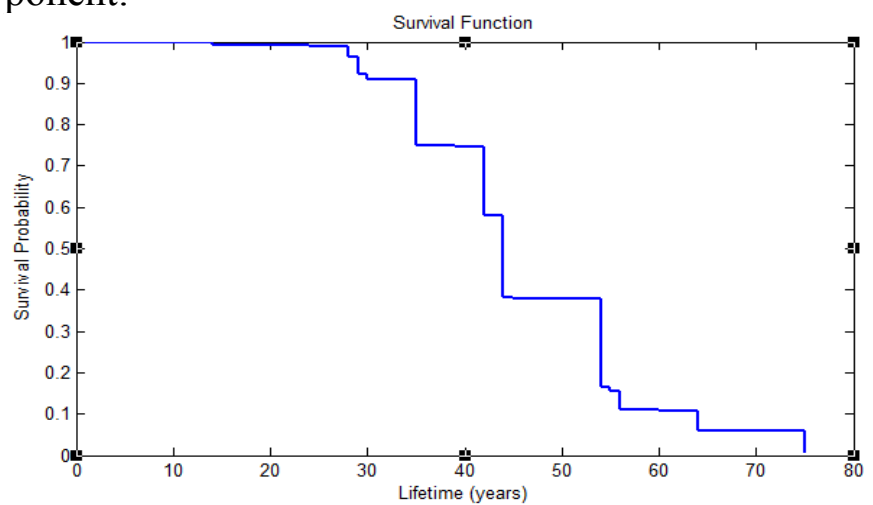

Figure 5. Survival function for the crossarms in a particular circuit

The survival probability in this case decreases faster. This is due to the fact that the wind speed in the area is lower than the average wind speed, and the difference in daily maximum and minimum temperatures is higher than the average for the whole sample. This component has over $80 \%$ chance of failing before 55 years of age.

Apart from the Survival function, the mean residual life $(m r l)$ per type of component/circuit was also obtained. To do so, a Weibull distribution was fitted to the data (failure times and censored times), as there is not a straightforward method to obtain this value with a Cox model. Figure 6 displays the fitting of the Weibull distribution to the Survival function for the whole sample.

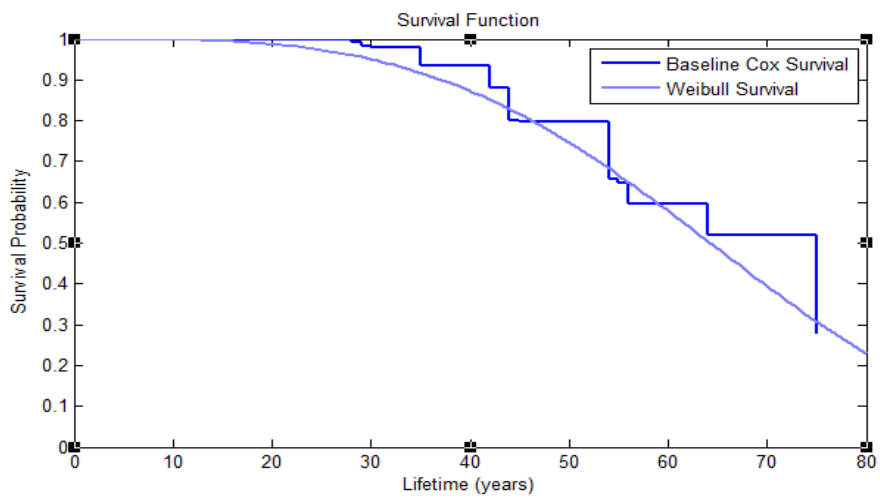

Figure 6. Cox and Weibull survival functions for the sample

Visually, the fitting of the curve seems to be a good approximation.

The $m r l$ is obtained by applying the following expression:

$m \hat{r}(t)=\frac{\int_{t}^{\infty} \bar{F}(x) d x}{\bar{F}(t)}=\frac{\int_{t}^{\infty} \exp \left\{-(x / \hat{\beta})^{\hat{\alpha}}\right\} d x}{\exp \left\{-(t / \hat{\beta})^{\hat{\alpha}}\right\}}$

where $t$ represents the age of the component, $\bar{F}(t)$ is the Weibull survival function at time $\mathrm{t} \hat{\alpha}$ and $\hat{\beta}$ are the estimated parameters of the Weibull distribution.

As an example of the calculation of the $\mathrm{mrl}$, if all the circuits in the sample are considered (Figure 6), the $\mathrm{mrl}$ of a component 30 years of age is 36 years. This value refers to an average tower/component of the sample. The $m r l$ can be calculated for specific circuits, however enough failure data from circuits with similar characteristics must be available.

Space constraints mean this cannot be covered in detail here but the residual life per component as well as the Survival curves can be used by the utility as a maintenance horizon, e.g. the outputs of the model can be utilised to increase inspection frequency in particular areas, or to plan in advance the replacement of certain components. 


\section{CONCLUSIONS AND FUTURE WORK}

This paper presents a Cox model to estimate the survival probability of steel tower components as well as the mean residual lifetime. Additionally, an indication of the increase in failure hazard per unit of increase of every covariate, i.e. temperature, wind speed, or magnetic field, is obtained. The outputs of the model allow for determining sites where components are at higher risk so that maintenance interventions can be prioritised, e.g. replacement of tower steelwork components or frequency of application of protective painting. Quality of steelwork from different years of installation could be also inferred.

The proposed model provides estimates of the tower steelwork component survival curves which experts agree to be reasonable. However, the information used to create the model brings uncertainty to the results. The Cox model is derived from information about the degradation state of steelwork components for a number of towers in a UK utility network. The assessment of the degradation of any component is obtained through visual inspection of the components and is given on a scale of 1 to 4 . It has been assumed here that the scale is indicating deterioration due to corrosion. However inspection reports provided by the utility show that this is not always the case, as bird activity, etc. is also factored into the condition assessment. Furthermore, at present a snapshot of the state of the components has been provided. Ideally, information on the state of the same components across different years should be taken into account, as well as the environmental conditions at the time. Finally, the proxy variables for the pollution concentration and airborne chloride concentration do not seem to explain the changes in deterioration of the steelwork components. It may be possible to take measurements or find better proxy variables for these factors in the future.

As possible future work, validation of the results and comparison with other data-driven techniques is of interest. Additionally, information regarding condition states 1 to 3 could also be considered in the model, as this information is currently excluded by the Cox model. As one approach, Cox models could be developed individually for the transition into state 2 and the transition into state 3 , in the same way as the current model predicts the transition into state 4 . Finally, the model could be extended to other components in the tower.

Acknowledgements The authors would like to thank the Centre for Environmental Data Analysis (CEDA) for providing access to climate data used in this project.

\section{REFERENCES}

British Standard ISO 9223. 2012 Corrosion of metals and alloys - Corrosivity of atmospheres - Classification, determination and estimation.

British Standard ISO 9224. 2012. Corrosion of metals and alloys - Corrosivity of atmospheres - Guiding values for the corrosivity categories.

Corder, G.W \& Foreman, D.I. 2009. Nonparametric statistics for non-statisticians - A step by step approach. John Wiley \& Sons.

Cox, D.R. 1972. Regression models and life tables. Journal of the Royal Statistical Society, Volume 34, No.2, pages: 187220.

Dean S.W \& Reiser D.B. 2002. Analysis of long term atmospheric results from ISO CORRAG program. Outdoor atmospheric corrosion, Townsend, H.E. Ed., USA, pages 318.

Feliu S; Morcillo, M \& Feliu Jr S. 1993. The prediction of atmospheric corrosion from meteorological and pollution parameters. Corrosion Science - I Annual Corrosion, Volume 34, No. 3, pages 403-414.

Feliu, S; Morcillo, M \& Chico, B.1999. Effect of distance from sea on atmospheric corrosion rate. Corrosion, Volume 55 , pages $883-891$.

Haynie, F.H. 1998. Environmental factors affecting the corrosion of galvanaised steel, degradation of metals in the atmosphere. Computer modelling in corrosion, Dean $\mathrm{S}$ and Le TS, editors, Philadelphia.

Kenny, E.D; Paredes, R; de Lacerda, L.A ; Sica, Y.C; de Souza, G.P \& Lazaris, J. 2009. Artificial neural network corrosion modelling for metals in an equatorial climate. Corrosion science, Volume 51, pages 2266-2278.

Klassen, R.D \& Roberge, P.R. 2001. The effects of wind on local atmospheric corrosivity. Corrosion 2001, NACE International.

Kleinbaum, D.G. \& Klein M. 2005. Survival analysis: A selflearning text. Springer.

Lyon, S. B; Wong, C.W \& Ajiboye, P. 1995. An approach to the modelling of atmospheric corrosion. Computer modelling in corrosion, Kirk WW and Lawson $\mathrm{HH}$, editors, Philadelphia.

Pintos, S; Queioi, N.V; de Rincon, O.T; Rincon, A \& Morcillo, M. 2000. Artificial Neural Network modelling atmospheric corrosion in the MICAT project. Corrosion Science, Volume 42, 2000, pages 35-52

Roberge, P.R; Klassen R.D \& Haberecht, P.W. 2002. Atmospheric corrosivity - a review. Materials and design, Volume 23, pages 321-330.

Straub, D \& Faber, M.H.2007. Temporal variability in corrosion modelling and reliability updating. Journal of Offshore Mechanics and Artict Engineering, Volume 129, pages 265-272.

Tidblad, J; Mikhilaov, A.A \& Kucera, V.2000. Application of a model for prediction of atmospheric corrosion in tropical environments. Marine Corrosion in Tropical Environments, Dean S, Hernandez-Duque Delgadillo G and Bushman JB editors, Philadelphia. 